\title{
EFFICIENT CALIBRATION OF DISCRETE ELEMENT MATERIAL MODEL PARAMETERS USING LATIN HYPERCUBE SAMPLING AND KRIGING
}

\author{
M. Rackl ${ }^{1}$, C.D. Görnig ${ }^{1}$, K.J. Hanley ${ }^{2}$ and W.A. Günthner ${ }^{1}$ \\ ${ }^{1}$ Institute for Materials Handling, Material Flow, Logistics \\ Technical University of Munich \\ Boltzmannstraße 15, 85748 Garching, Germany \\ e-mail: rackl@fml.mw.tum.de,guenthner@fml.mw.tum.de \\ ${ }^{2}$ Institute for Infrastructure and Environment, School of Engineering \\ The University of Edinburgh \\ Edinburgh EH9 3JL, United Kingdom \\ e-mail: K.Hanley@ed.ac.uk
}

Keywords: discrete element method, calibration, meta model, Kriging

\begin{abstract}
Material model parameter identification for discrete element models (DEM) is typically done using a trial-and-error approach and its outcome depends largely on the experience of the DEM user. This paper describes a work flow which facilitates the efficient and systematic calibration of discrete element material models against experimental data. The described workflow comprises three steps. In the first step, an approach based on the design and analysis of computer experiments (DACE) is adopted in which data is generated for the parametrisation of Kriging models based on Latin hypercube sampling. In the second step, multi-objective optimisation is applied to the Kriging models. This study introduces an additional cost criterion, which includes the Rayleigh time step, in order to reduce the solution set size to one element. In the third step, the optimisation procedure is repeated with the actual DEM models, using the optimal parameter set from the Kriging models as the start value. This final step with the full DEM models refines the parameter set against experimental data. Since DEM material model calibration is time-consuming, the workflow is implemented into an automated process chain. In this paper, the methodology is described in detail and results are shown which illustrate the usefulness and effectiveness of this approach. Initial verification simulations run using the calibrated parameters give good agreement with experimental results.
\end{abstract}




\section{Introduction}

Many advanced modelling methods and tools are available, and the continual increases in affordable computational power allow more ambitious and realistic simulations to be run yearon-year. One remaining problem affecting certain simulation methods, which particularly inhibits their adoption in industry, is the difficulty in choosing suitable input parameters. This is especially true when the simulation input parameter does not have a physical analogue.

This paper focuses on one simulation tool, the discrete element method (DEM). A work flow is described and demonstrated for calibration of key simulation input parameters against experimental data. The objective is to allow DEM users to identify their required input parameters in an efficient, user-friendly and systematic manner.

\subsection{Overview of discrete element method}

Since its formulation in the 1970s [1], the discrete element method (DEM) has become increasingly popular for simulating complex systems of particulates at the particle scale by specifying a relatively small number of microstructural parameters. The growing interest in DEM among the scientific community is demonstrated by the publication of a number of special issues of journals devoted to DEM within the past five years (e.g., Engineering Computations 26(6); Granular Matter 11(5); Powder Technology 193(3); Powder Technology 248). Literature surveys have also shown a rapid increase in the popularity of DEM since its introduction $[2,3]$.

In 'soft-sphere' DEM simulations, particles are modelled as bodies of finite size, inertia and stiffness. The geometries of the simulated particles are usually idealised to reduce the computational requirements. The particles are rigid but are allowed to overlap; this interparticle overlap is analogous to the deformation that occurs at real particle contacts. The most common implementation of DEM is based on an explicit, conditionally-stable time-stepping algorithm. In each time step [3], interparticle forces are evaluated at contact points and resultant forces are calculated for each particle. Newton's Second Law is then applied to determine the translational and angular particle accelerations. These acceleration terms are integrated numerically to find particle velocities and displacements. Finally, the displacements are used to update the particle positions during each time step.

\subsection{DEM material parameter identification}

Even though a typical DEM simulation requires relatively few input parameters, finding these can be challenging. Consider the simplest case in which each idealised, simulated particle represents one physical particle. The rheological parameters for input to the contact models (i.e., force-displacement laws) can be difficult to determine accurately by experiment [4]. The interparticle friction coefficient strongly influences the system response in simulations using spheres [5], but its value is often increased beyond physical measurements of friction in an effort to model the particle irregularity in the real system. Numerical damping [6,7] is difficult to relate to physical measurements. These problems are exacerbated when irregular particles are simulated: even simple rolling resistance models require at least one non-physical parameter [8]. Other complications of the basic DEM, e.g., crushable fundamental particles, also require parameters which lack a physical basis [9]. 'Coarse graining' may be used to reduce the computational requirements of a simulation. The real particles are replaced by fewer, larger coarse particles, each of which represents many real particles $[10,11,12]$. Thus, none of the input parameters for a coarse-grained simulation may necessarily be obtainable by laboratory testing.

In general, it is not possible to infer a complete set of appropriate parameters for a DEM 
simulation directly from measured properties of the physical material. Therefore, some (or all) of the parameters may need to be determined by calibration, i.e., by varying the unknown DEM parameters until a good match is obtained between physical measurements of the response(s) of interest and the simulation results. Although DEM calibration is often used, e.g., [13, 14, 15], the main limitation of existing, commonly-used calibration methods is inefficiency. The parameters are often varied individually by trial-and-error while the user-defined response of interest is monitored. This approach has many disadvantages [4]: the effectiveness of the approach is dependant on the experience of the user, the final parameters obtained may not be optimal, the number of DEM simulations required for calibration is not known in advance, and limited mechanistic insight is gained. As DEM simulations can be very computationally expensive, it is desirable to maximise the efficiency of the calibration process. Several researchers have proposed more efficient DEM calibration approaches using design of experiments (DoE) methods $[4,16,17,18]$ but these have found limited application to date. In subsection 2.4 of this paper, an alternative work flow for DEM calibration is described which is both efficient and user-friendly.

\section{MATERIALS AND METHODS}

The work flow proposed in this paper is based on Kriging meta modelling, multi-variate optimisation and general concepts of Design and Analysis of Computer Experiments. A detailed description of the process implemented using GNU Octave (Octave, [19]) is given in subsection 2.4 .

\subsection{Design and Analysis of Computer Experiments}

Design and Analysis of Computer Experiments (DACE) is closely related to the Design of Experiments (DoE) for physical experiments. It includes methods and work flows for the characterisation of computationally inexpensive and expensive black box models. In the case of DEM material parameter calibration, the black box models are the material test setups, which were modelled using DEM. The reader is referred to widely-available literature on DoE and DACE for nomenclature, general ideas as well as background of the methods (e. g. [20, 21]).

As with DoE, any DACE workflow can be divided into four steps:

1. Define the factors, factor levels (or factor intervals) and the response to be recorded.

2. Create an experimental plan based on the data of step 1 and the requirements for step 4.

3. Conduct the experiments by running simulations according to the experimental plan and gather the response data.

4. Transform (where required) the data, use them to parametrise meta models and harness the latter for e.g. optimisation of the response.

\subsection{Kriging and Latin Hypercube sampling}

Kriging, which is also known as Gaussian process regression, is a statistical regression method for meta modelling which is based on covariances. It requires response data from several samples taken from within a previously-defined $\mathrm{N}$-dimensional factor space and estimates the (meta) model parameters via a maximum likelihood approach [22]. Basic ideas for Kriging were developed and applied in [23] and later generalised [24]. In this study, the Kriging 
implementation of the Small Toolbox for Kriging (STK, [25]) was used under Octave. The baseline options for the Kriging models were set to using an anisotropic Matérn covariance matrix together with a linear trend.

The experimental plan, which prescribes the factor value combinations at which the response data are to be computed, is generated via Latin hypercube sampling (LHS, [26]). LHS generates factor value combinations by randomly, but evenly, distributing points within the specified factor interval limits. To further reduce the variance of the Kriging models, this study uses LHS in conjunction with a MAXIMIN criterion [21, p. $138 \mathrm{ff}$.].

\subsection{Issues addressed by the proposed work flow}

Since the calibration of DEM material parameters is greatly dependent on the DEM user, the aim of the proposed work flow was to reduce the user interaction to a minimum. Three major issues with DEM material calibration were selected to be addressed by the work flow.

First of all, the calibration process should become automatic, which means that computations required to calibrate the material parameters are to be set up and run automatically. This also includes the evaluation of DEM results. While the manual evaluation of e.g. the bulk density is fairly easy, the measurement of, for example, the angle of repose is somewhat subjective and its result may vary with the measurement method used. It is therefore important to ensure comparability of different values of the same response, which is solved using an automatised results evaluation.

Secondly, keeping track of how changes of the material parameters affect the DEM results is a challenging task, even for an experienced user. Due to the sheer number of up to 14 parameters ${ }^{1}$ and possible interactions, it becomes very difficult to relate parameters to effects when altering several parameters at once. This problem could be resolved by only changing one factor at a time; however this approach does not account for parameter interactions and is highly inefficient [20]. The solution suggested in this study is to combine LHS and Kriging methods to create meta models that account for interactions and corresponding effects.

Thirdly, the computational efficiency of the calibrated material model may not always be the focus of the calibration process. This is especially true since the Rayleigh time step in DEM depends on material parameter values (i. e. altering the Young's modulus affects the maximum time step size). Hence, a further target of the described work flow is to take the Rayleigh time step into account and not only seek precise prediction of measurement results but also larger minimum time step sizes. Within subsection 2.4, a cost function that accounts for the Rayleigh time step is proposed and implemented.

\subsection{Work flow of the proposed calibration process}

The described workflow was developed and implemented using the open-source DEM software LIGGGHTS [29]. Since DEM material model calibration is time-consuming, the workflow is implemented into an automated process chain using GNU Octave. The user has to integrate custom DEM models of material tests into the folder structure of the Octave scripts. A custom DEM model usually consists of a LIGGGHTS model of a bulk material test along with auxiliary functions which allow for automatic evaluation of the result files. For example, this could be the model of a simple bulk density experiment, including Octave functions to compute the bulk density from the total particle mass and the known volume of the container ${ }^{2}$.

\footnotetext{
${ }^{1} \mathrm{DE}$ model with one particulate medium and wall interaction.

${ }^{2}$ Setting up custom models is a non-recurring task.
} 
The only actual user input are the measurement results from physical experiments (e. g. bulk density) and intervals for the material and contact parameters of materials in Octave. These intervals will have to include the final solution set. Of course, the final solution set is unknown at this point, so the intervals can be of any finite width. Subsequently, the algorithm can be started and it will finish autonomously.

The main workflow comprises three steps. In the first step, an approach based on the design and analysis of computer experiments is adopted in which data is generated for the parametrisation of Kriging models based on Latin hypercube sampling. The material and contact parameter values, as defined by the user, are used as free variables for the calibration. The programme will carry out a user-specified number of DEM simulations, which provide the input for the Kriging meta model parametrisation (cf. Figure 1). Depending on the performance of the computer, several simulations may be run in parallel to save time. All obtained results are handed over and saved in Octave.

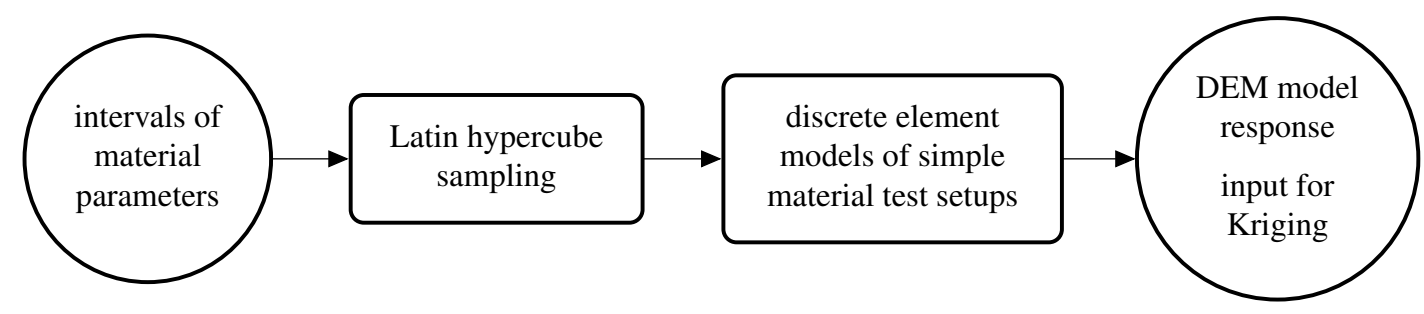

Figure 1: Generation of response data as input for the Kriging models.

In the second step, multi-objective optimisation is applied to the Kriging models (cf. Figure 2). For the optimisation the frontend 'nonlin-residmin' Octave function for non-linear residual minimization is used which is based on the Levenberg-Marquardt algorithm [27, 28]. All results of the previous DEM simulations and the Latin hypercube sampling plan are used as responses to parametrise Kriging models. The Kriging models are then used to optimise the set of variable material and contact parameters such that the user-specified measurement values are attained.

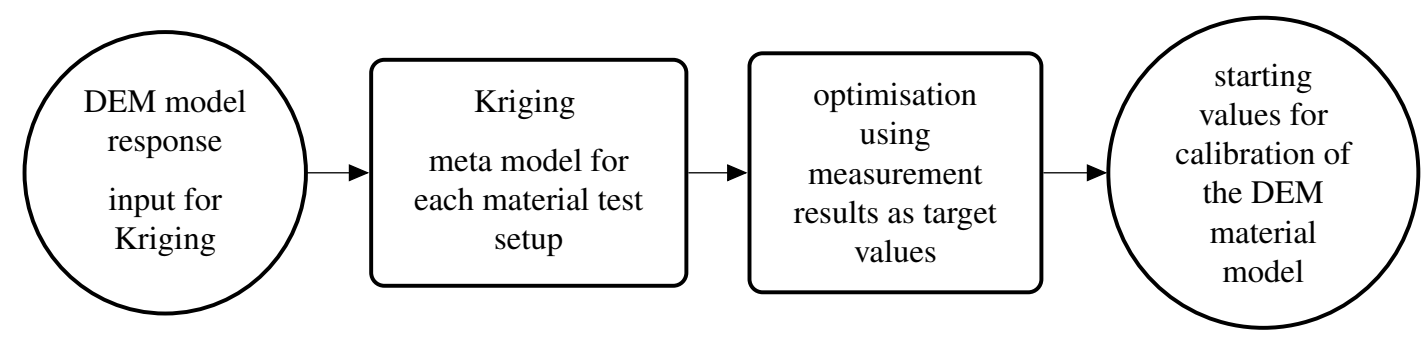

Figure 2: Response data from the LHS are used as input to the Kriging models.

For this purpose, the multi-objective cost function

$$
c_{i}=\frac{s_{i}-m_{i}}{m_{i}}
$$

has been implemented where $s_{i}$ is the result of the simulation and $m_{i}$ is the corresponding physical measurement. The smaller the difference between the two, the smaller $c_{i}$ is. The function calculates $i$ proportions of $i$ measurement results and all have the same weighting. To avoid generating computationally-expensive sets of DEM parameters, the Rayleigh time step 
will also be optimised. The cost function of the Rayleigh time step is

$$
t_{i}=\frac{r_{\max }-d t_{r}}{r_{\max }-r_{\min }}
$$

where $d t_{r}$ is the Rayleigh time step, $r_{\max }$ is the best-case of the Rayleigh time step and $r_{\min }$ is the worst-case. Best and worst-case refer the minimum and maximum achievable Rayleigh time with respect to the intervals specified. The Rayleigh time step is calculated according to

$$
d t_{r}=\pi \cdot r \cdot \frac{\sqrt{\frac{\rho}{G}}}{0.1631 \cdot \nu+0.8766},
$$

where $\rho$ is particle density, $G$ is the shear modulus, $\nu$ is Poisson's ratio and $r$ is the radius of the smallest particle. The Rayleigh time step depends on the parameter values that have to be optimised. The cost function of the Rayleigh time step is linear and it can take values between 0 and 1.

In the third step, the optimisation procedure is repeated with the actual DEM models, using the optimal parameter set from the Kriging models as start values (cf. Figure 3). This final step only requires a few more runs with the full DEM models and simultaneously refines the parameter set against the experimental measurement data.

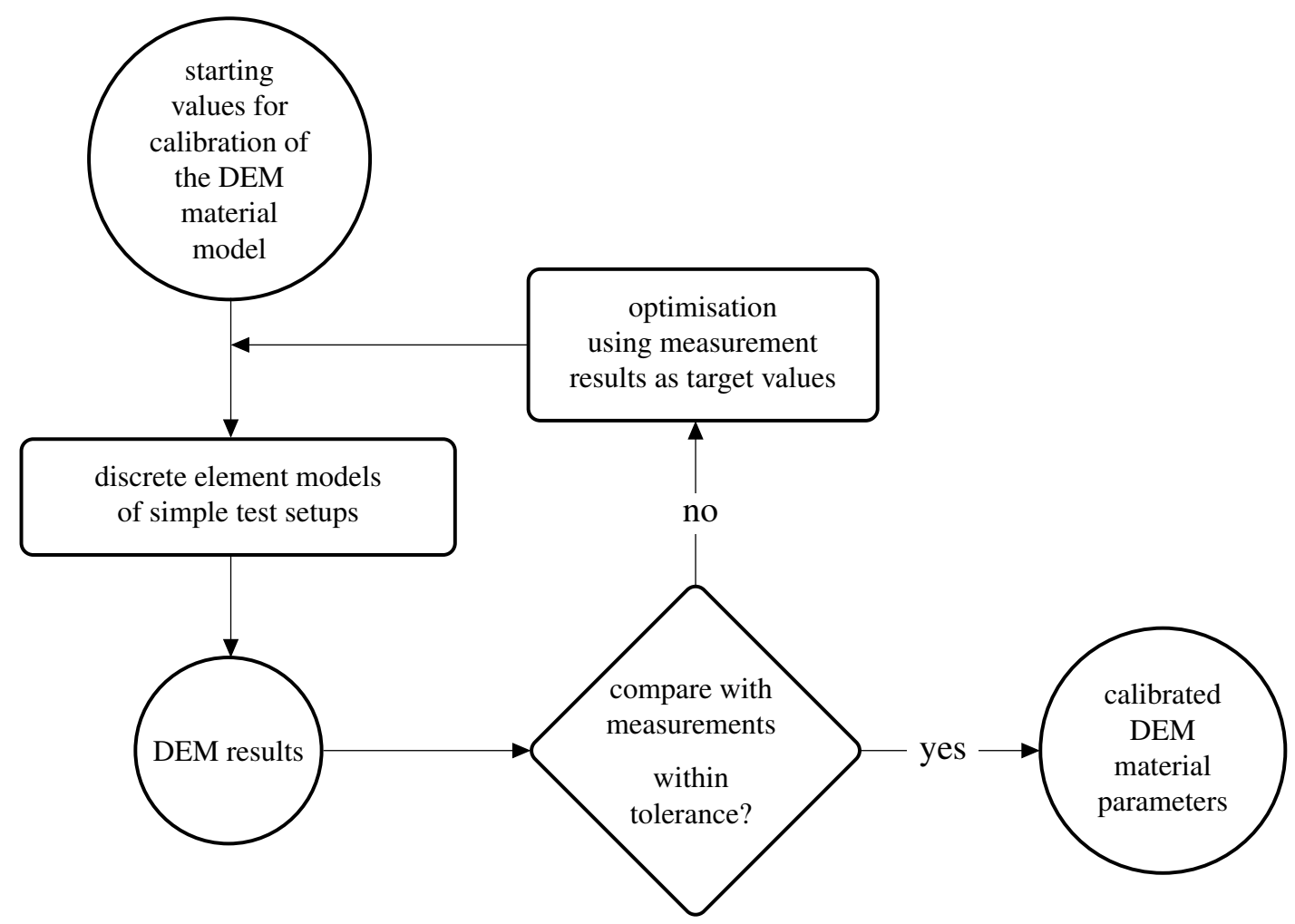

Figure 3: Optimisation procedure for DEM models

\subsection{A small study using glass beads}

A small initial study was set up in order to test the proposed work flow within a realistic framework and glass beads were chosen as the bulk solid of interest. The overall aim was to 
calibrate the density and rolling friction (particle-particle) in such way that the angle of repose and bulk density results match measured values. A bulk density of $1500 \mathrm{~kg} \mathrm{~m}^{-3}$ and an angle of repose of $22^{\circ}$ were found in literature for glass beads ([30, p. 16], [31]). The particle diameter was set to $10 \mathrm{~mm}$.

A combined discrete element model was used for simulation of the bulk density and angle of repose. Since measuring the angle of repose can be difficult, an automatic evaluation algorithm, based on image recognition, was developed for this study. The DEM software LIGGGHTS was applied with a Hertz-Mindlin contact model and the elastic-plastic spring-dashpot (EPSD) rolling friction model. Table 1 shows the DEM input parameters used.

\begin{tabular}{llllll}
\hline material parameter & particle & wall & p-p & p-w & constant? \\
\hline Young's modulus $\left(\mathrm{N} \mathrm{m}^{-2}\right)$ & $3.6 \times 10^{5}$ & $2.11 \times 10^{11}$ & & & yes \\
Poisson's ratio (-) & 0.22 & 0.30 & & & yes \\
density $\left(\mathrm{kg} \mathrm{m}^{-3}\right)$ & 2100 & 7700 & & & used for calibration \\
\hline contact parameter & & & & & \\
\hline friction coefficient (-) & & & 0.18 & 0.27 & yes \\
rolling friction (-) & & & 0.67 & 0.005 & used for calibration \\
coefficient of restitution (-) & & & 0.79 & 0.82 & yes \\
viscous RF damping (-) & & & 0.25 & 0.25 & yes \\
\hline
\end{tabular}

Table 1: Constant DEM input parameters and those used for calibration of the bulk density and angle of repose (p-p: particle-particle; p-w: particle-wall; RF: rolling friction).

In addition to running the described test case, the influence of the factor interval width (FIW) and the random factory seed (RFS) of the DEM models was investigated for the purpose of testing the robustness of the work flow. The FIW prescribes how wide the factor interval of the calibration parameters is, whereas the RFS controls the random generation of particles when starting a DEM simulation in LIGGGHTS. The influence of RFS on the results should only be of a stochastic nature, in theory. Table 2 shows details about the RFS and FIW levels and the experimental plan for this study. For the two non-constant parameters-density and rolling friction- maximum and minimum values (intervals) were specified before each calibration run $( \pm$ FIW). During each calibration run 26 probes were computed to parametrise the Kriging models.

\begin{tabular}{ccc}
\hline & FIW $(\%)$ & RFS $(-)$ \\
levels & $10,33,66$ & 3000,4000 \\
\hline run no. & & \\
1 & 10 & 3000 \\
2 & 10 & 4000 \\
3 & 33 & 3000 \\
4 & 33 & 4000 \\
5 & 66 & 3000 \\
6 & 66 & 4000
\end{tabular}

Table 2: Factor levels and experimental plan for the study to investigate factor interval width (FIW) and random factory seed (RFS).

\section{RESULTS AND DISCUSSION}

Two factors, the FIW and the RFS, were altered to initially assess the reliability of the work flow for DEM material parameter calibration. Each of the runs from the experimental plan 
converged to a meaningful solution set. It took the multi-variate optimisation algorithm a mean of 10 optimisation runs (min. 9, max. 15) with the actual DEM models.

The desired values for bulk density and angle of repose were closely met, as deviations were within a few percent. Mean differences were $1.3 \%$ ( $\min .0 .45 \%$, max. $2.7 \%$ ) for the angle of repose and $0.92 \%$ (min. $0.13 \%$, max. $3.1 \%$ ) for bulk density. One should also note that the variance measured in physical experiments, especially for the angle of repose, is typically significantly higher than the deviations recorded in this study.

Due to the small number of simulation experiments, it is not fully clear how much the RFS value influences the results. Ideally, it should only induce stochastic noise. According to the results depicted in Figure 4 and Figure 5, it does not generate a systematic trend or offset.

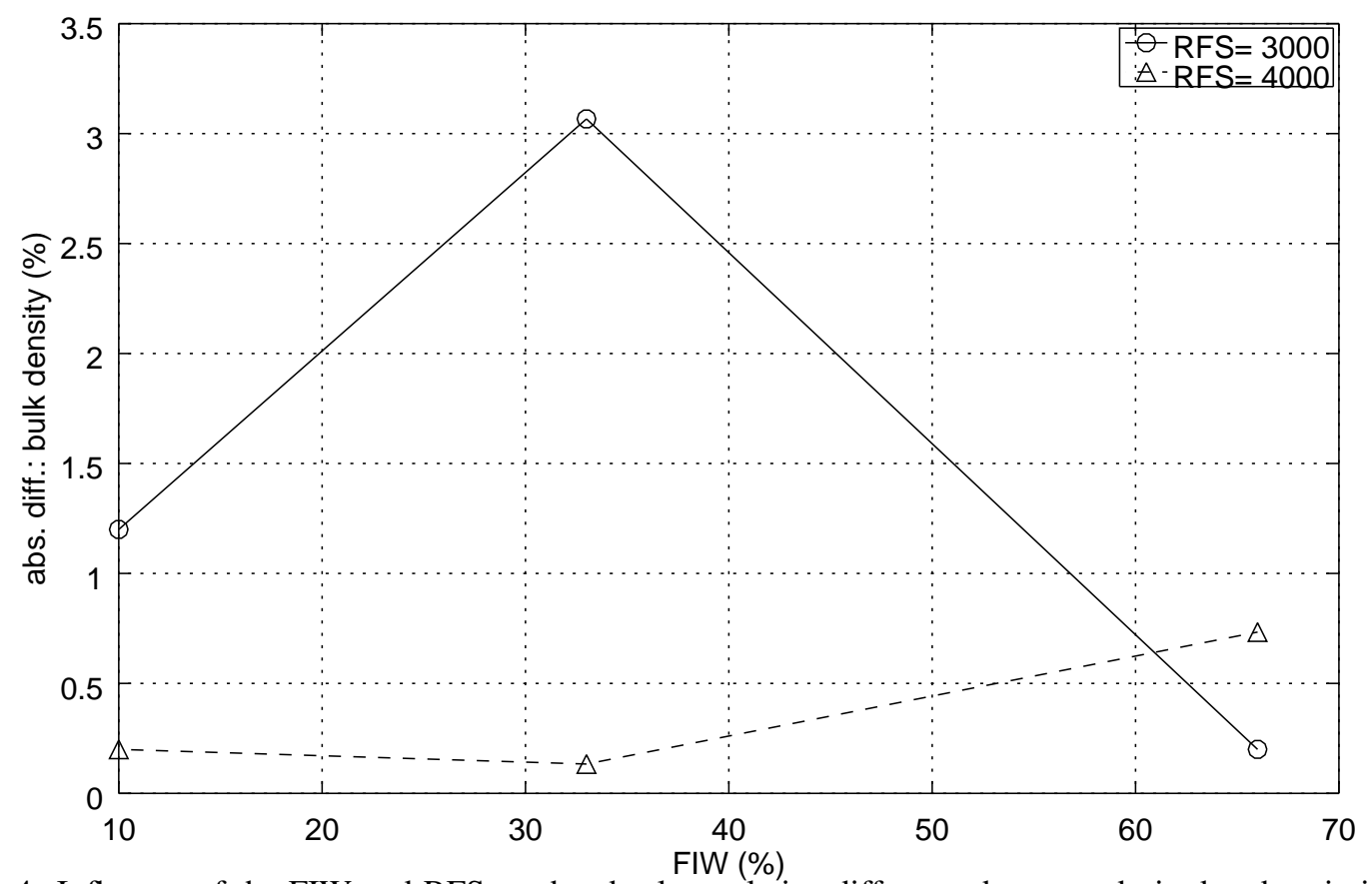

Figure 4: Influence of the FIW and RFS on the absolute relative difference between desired and optimised bulk density.

There is a clear correlation between the number of optimisation runs and the FIW. It can be seen from Figure 6 that a wider FIW results in more optimisation runs being required, independently of the RFS. This behaviour was expected, since the sampling density reduces with an increased FIW and the Kriging models have to interpolate in areas with a greater distance between interpolation points. The Kriging model predictions therefore show a greater variance on those areas and the precision of the estimates for starting values for the DEM optimisation reduces.

\section{CONCLUSION}

A framework for efficient calibration of discrete element material model parameters was implemented in this study. A total of approximately 38 DEM runs were required to calibrate particle density and rolling friction against measurements of the angle of repose and bulk density. The results from a small study with glass beads show that the general approach works and desired results were well within practical tolerances of the desired values. Nonetheless, more studies will be necessary to further validate the usefulness of the described work flow as well as its robustness, e. g. when unfavourable parameter intervals are set by the user. 


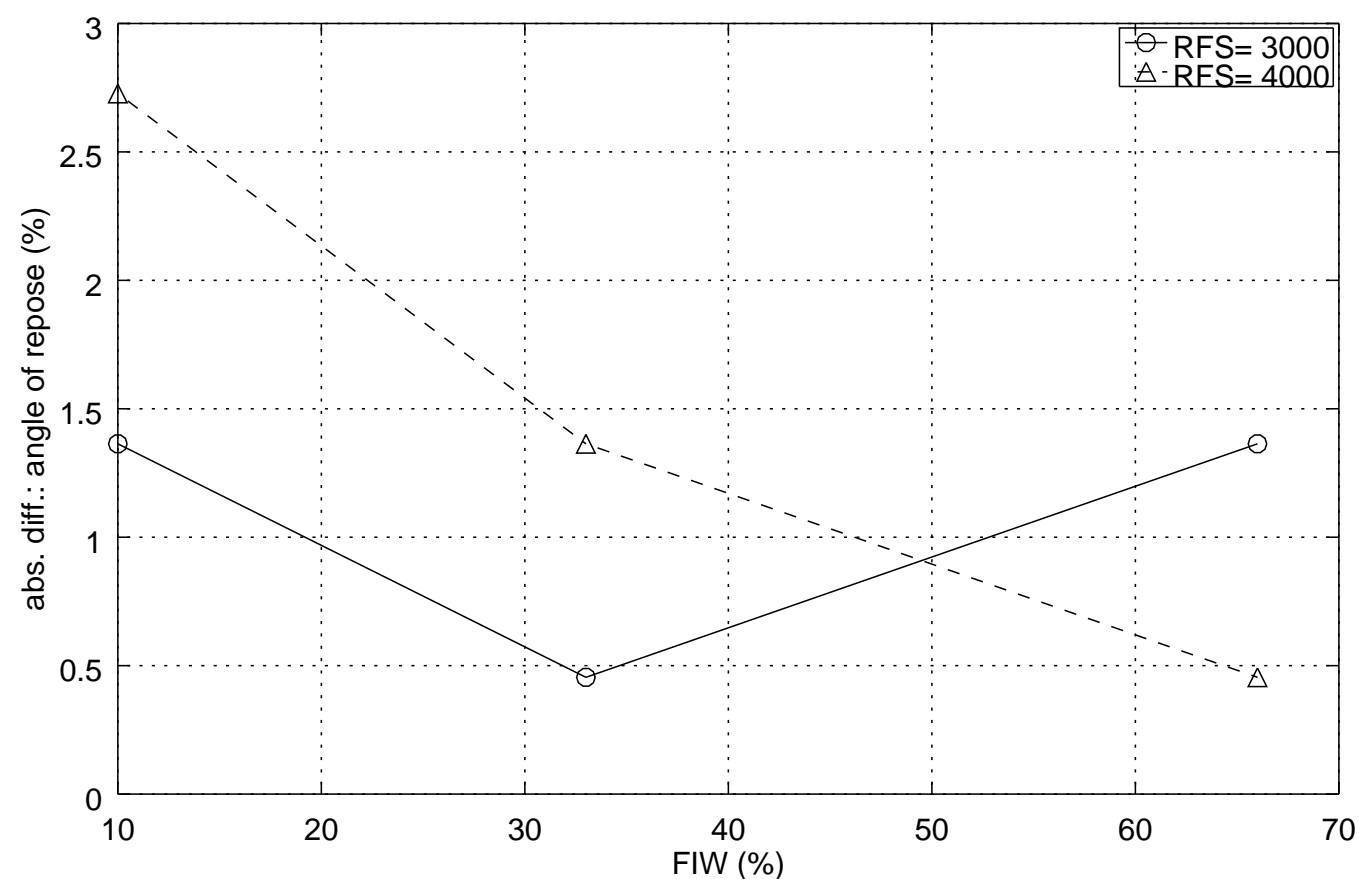

Figure 5: Influence of the FIW and RFS on the absolute relative difference between desired and optimised angle of repose.

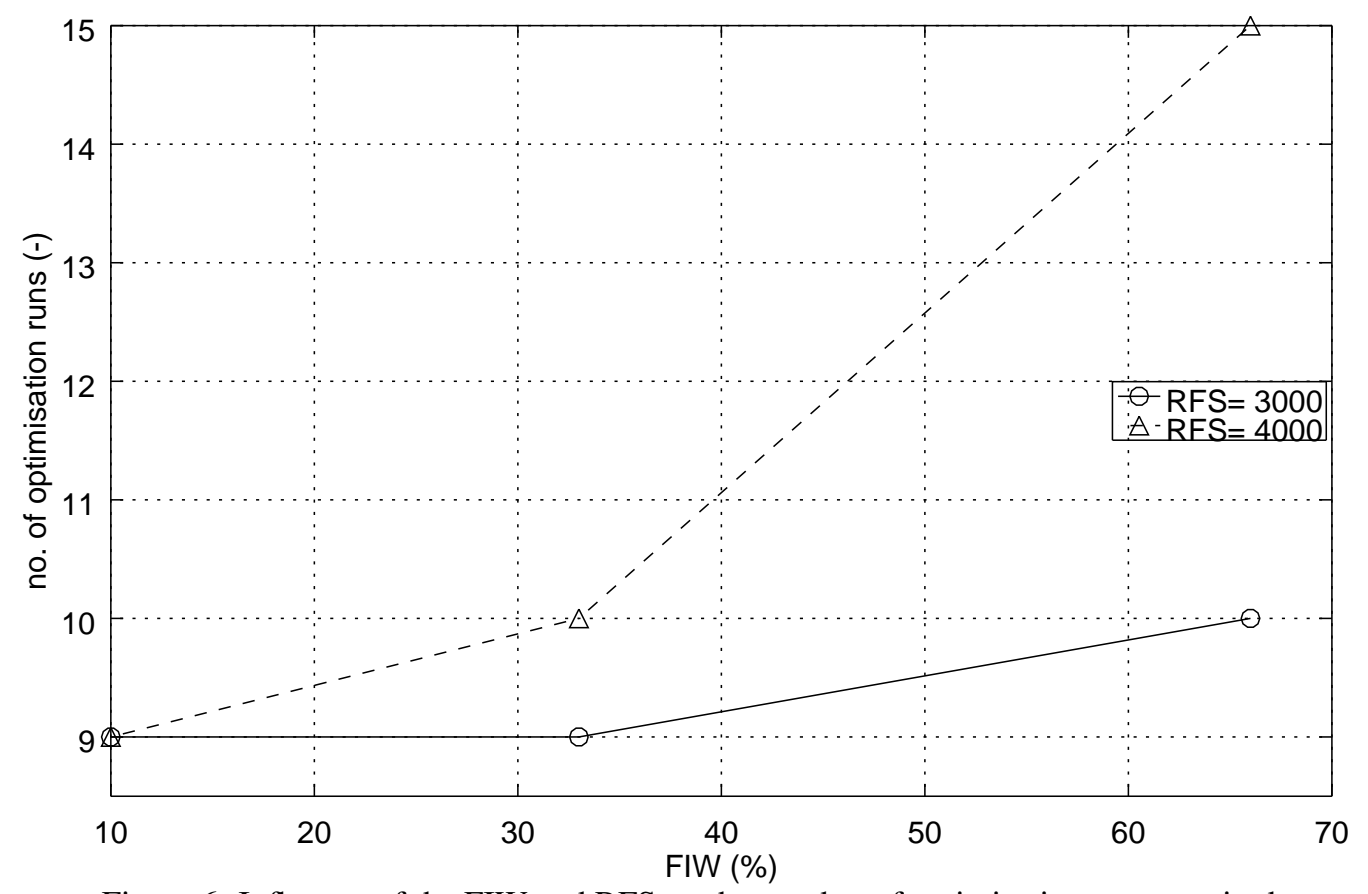

Figure 6: Influence of the FIW and RFS on the number of optimisation runs required. 


\section{ACKNOWLEDGEMENT}

This study received funding by the AiF (no. 18371 N/1), within the programme for sponsorship by Industrial Joint Research (IGF) of the German Federal Ministry of Economic Affairs and Energy based on an enactment of the German Parliament.

\section{REFERENCES}

[1] Cundall, P.A. \& Strack, O.D.L. (1979). A discrete numerical model for granular assemblies. Géotechnique, 29(1): 47-65.

[2] Zhu, H.P., Zhou, Z.Y., Yang, R.Y. \& Yu, A.B. (2007). Discrete particle simulation of particulate systems: Theoretical developments. Chemical Engineering Science, 62(13): 3378-3396.

[3] O’Sullivan, C. (2011). Particulate discrete element modelling: A geomechanics perspective ( $1^{\text {st }}$ ed.), Taylor \& Francis, Oxford, UK.

[4] Hanley, K.J., O’Sullivan, C., Oliveira, J.C., Cronin, K. \& Byrne, E.P. (2011). Application of Taguchi methods to DEM calibration of bonded agglomerates. Powder Technology, 210(3): 230-240.

[5] Huang, X., Hanley, K.J., O’Sullivan, C. \& Kwok, C.-Y. (2014). Exploring the influence of interparticle friction on critical state behaviour using DEM. International Journal for Numerical and Analytical Methods in Geomechanics, 38(12): 1276-1297.

[6] Potyondy, D.O. \& Cundall, P.A. (2004). A bonded-particle model for rock. International Journal of Rock Mechanics and Mining Sciences, 41(8): 1329-1364.

[7] Itasca Consulting Group (2008). PFC3D: Particle flow code in three dimensions User's Guide $\left(4^{\text {th }}\right.$ ed.), Minneapolis, Minnesota.

[8] Ai, J., Chen, J.F., Rotter, J.M. \& Ooi, J.Y. (2011). Assessment of rolling resistance models in discrete element simulations. Powder Technology, 206, 269-282.

[9] Hanley, K.J., O’Sullivan, C. \& Huang, X. (2015). Particle-scale mechanics of sand crushing in compression and shearing using DEM. Soils and Foundations, 55(5): 1100-1112.

[10] Sakai, M. \& Koshizuka, S. (2009). Large-scale discrete element modeling in pneumatic conveying. Chemical Engineering Science, 64(3): 533-539.

[11] Sakai, M., Abe, M., Shigeto, Y., Mizutani, S., Takahashi, H., Viré, A., Percival, J.R., Xiang, J. \& Pain, C.C. (2014). Verification and validation of a coarse grain model of the DEM in a bubbling fluidized bed. Chemical Engineering Journal, 244: 33-43.

[12] Nasato, D.S., Goniva, C., Pirker, S. \& Kloss, C. (2015). Coarse Graining for Large-scale DEM Simulations of Particle Flow - An Investigation on Contact and Cohesion Models. Procedia Engineering, 102: 1484-1490.

[13] Doležalová, M., Czene, P. \& Havel, F. (2002). Micromechanical modeling of stress path effects using PFC2D code. In: H. Konietzky (Ed.), Numerical Modeling in Micromechanics via Particle Methods, Balkema, Lisse, pp. 173-182. 
[14] Asaf, Z., Rubinstein, D. \& Shmulevich, I. (2006). Evaluation of link-track performances using DEM. Journal of Terramechanics, 43(2): 141-161.

[15] Coetzee, C.J. \& Els, D.N.J. (2009). Calibration of discrete element parameters and the modelling of silo discharge and bucket filling. Computers and Electronics in Agriculture, 65(2): 198-212.

[16] Yoon, J. (2007). Application of experimental design and optimization to PFC model calibration in uniaxial compression simulation. International Journal of Rock Mechanics and Mining Sciences, 44(6): 871-889.

[17] Favier, J., Curry, D. \& LaRoche, R. (2010). Calibration of DEM material models to approximate bulk particle characteristics. Proc. $6^{\text {th }}$ World Congress on Particle Technology, Nuremberg, Germany.

[18] Johnstone, M. (2010). Calibration of DEM models for granular materials using bulk physical tests. PhD thesis, University of Edinburgh.

[19] Eaton, J.W., Bateman, D., Hauberg, S., Wehbring, R. (2015). GNU Octave version 4.0.0 manual: a high-level interactive language for numerical computations.

[20] Dean, A., Morris, M., Stufken, J., and Bingham, D. (2015). Handbook of Design and Analysis of Experiments. CRC Press, Hoboken.

[21] Santner, T.J., Williams, B.J., Notz, W.I (2003). The Design and Analysis of Computer Experiments. Springer Series in Statistics. Springer, New York, NY.

[22] Jones, D.R., Schonlau, M., Welch, W.J. (1998). Efficient global optimization of expensive black-box functions. Journal of Global Optimization, 13(4):455-492.

[23] Krige, D.G. (1951). A statistical approach to some mine valuations and allied problems at the Witwatersrand. Master's thesis, University of Witwatersrand.

[24] Matheron, G. (1963). Principles of geostatistics. Economic Geology, 58(8):1246-1266.

[25] Bect, J., Vazquez, E. et al (2014). STK: a Small (Matlab/Octave) Toolbox for Kriging. Release 2.3.

[26] McKay, M.D., Beckman, R.J., Conover, W.J. (1979). A comparison of three methods for selecting values of input variables in the analysis of output from a computer code. Technometrics, 21(2):239.

[27] D.W. Marquardt. (1963). An algorithm for least-squares estimation of nonlinear parameters. Journal of the Society for Industrial and Applied Mathematics, 11(2):431-441.

[28] K. Levenberg. (1944). A Method for the Solution of Certain Non-Linear Problems in Least Squares. The Quarterly of Applied Mathematics, 2: 164-168.

[29] Kloss, C., Goniva, C., Hager, A., Amberger, S., Pirker, S. (2012). Models, algorithms and validation for opensource DEM and CFD-DEM. Progress in Computational Fluid Dynamics, 12(2/3):140-152. 
[30] Brown, R.L., Richards, J. C., Danckwerts, P. V. (1970). Principles of Powder Mechanics: Essays on the Packing and Flow of Powders and Bulk Solids, volume volume 10 of International series of monographs in chemical engineering. Elsevier Science, Burlington.

[31] Wong, A.C.-Y. (2000). Characterisation of the flowability of glass beads by bulk densities ratio. Chemical Engineering Science, 55(18):3855-3859. 